\title{
XXIII.
}

\section{Ueber das Verhalten der äusseren Körnerschicht der Netzhaut bei gewissen Krankheiten.}

\author{
Von Dr. Gabriel Denissenko \\ aus St. Petersburg. \\ (Aus dem Laboratorium des pathologischen Instltuts zu Berlin.)
}

(Hierzu Taf. IX. Fig. 1-3.)

Sämmtliche Veränderungen, die man bei der Bright'schen Krankheit wahrgenommen hat, lassen sich zurückführen 1) auf eine Stauungshyperämie in der Netzhaut, 2) auf den Austritt von Flüssigkeit aus den Gefässen in das Gewebe der Netzhaut. Durch diese Ursachen entstehen in dem letzteren grosse Veränderungen, die von den Ophthalmologen und Pathologen schon längst erkannt und beschrieben worden sind. Es ist bekannt, dass die Netzhaut bei dieser Krankheit stellenweise verdickt wird und ibre Oberfläche ein wellenförmiges Aussehen bekommt; es ist ferner bekannt, dass es innerhalb der Netzhaut, einschliesslich der äusseren Körnerschicht, zu bedeutenden Hämorrhagien bommt. Alles dieses deutet auf einen, in der Netzhaut herrschenden bedeutenden Druck- und starken Spannungsunterschied; dennoch wird vor allen Untersuchern angegeben, dass in dem Gewebe der äusseren Körnerschicht keine Veränderungen zu bemerken waren.

Mir ist es immer unwahrscheinlich erschienen, dass solch ein starker Blutdruck, wie er in der Netzhaut beim Morbus Brightii herrscht, ein Druck, der zu fast makroskopischen Veränderungen in derselben führt, ganz ohne Einfluss auf die äussere Körnerschicht bleiben sollte.

Herr Prof. Virchow hat mir einige Augen von an Morbus Brightii verstorbenen Personen gütigst zur Verfügung gestellt. Diese Augen wurden erst mit Müller'scher Flüssigkeit behandelt, dann auf einen Tag in Wasser gelegt, aus dem Wasser brachte ich sie zuerst in Spiritus, dann in Alkohol. Aus der in dieser Weise gehärteten 
Netzhant schnitt ich runde Stückchen aus der Gegend der Macula lutea aus, klemmte sie in eine Amyloidmilz und führte einen Schnitt aus. Die Präparate färbte ich mit Hämatoxylin, nicht selten auch mit Eosin und untersuchte in Glycerin.

An solchen Präparaten fand sich, dass der in der Netzhaut verlaufene Krankheitsprozess wirklich auch in der äusseren Körnerschicht tiefe Spuren hinterlassen hatte ${ }^{x}$ ). Untersucht man eine in der angegebenen Weise bereitete Netzhaut bei schwacher Vergrösserung (Hartnack No. 4-5), so findet man folgende Veränderung: 1) die Memb. limit. ext. erscheint wie getrübt. 2) die Körner liegen von der Memb. limit. ext. etwas entfernt, so dass ein freier Zwischenraum zwischen ihnen und der Membran entsteht. 3) die Körner liegen auch von einander mehr entfernt, als im normalen Zustande. Untersuchen wir bei starker Vergrösserung, so finden wir, dass die Memb. limit., die im normalen Zustande eine homogene, stark lichtbrechende Haut darstellt, jetzt matt und schwach lichtbrechend geworden ist. Dieses rührt von Eindrücken oder Ausbuchtungen her, die sich auf ihrer inneren Fläche finden. Dieselben sind übrigens so schwach, dass sie nur bei sehr starker Vergrösserung (No. 9 trocken) und an ganz feinen Schnitten zu sehen sind. Auch wird durch diese Vertiefungen die Ebene der Membran wenig verändert. Die Stăbchen und die Zapfen bleiben in der äusseren Körnerschicht unverändert [Virchow $\left.\left.{ }^{2}\right)\right]$. Die Körner, welche im normalen Zustande beim Menschen hart an der Memb. limit. liegen ${ }^{3}$ ), sind bei der Bright'schen Krankheit etwas von

1) Da ich mir nlcht die Besprechung und Untersuchung der Bright'schen Krankheit zar Aufgabe gemacht babe, sondern nur einige bisher unbekannte Veranderungen beschreiben und ihre Ursachen erörtern wollte, so konnte ich auch nicht näher auf die reichbaltige Literatur über diese Krankheit eingehen, obgleich sich Männer mit sehr klangvollen Namen viel mit dieser Krankheit beschäftigt haben, wie Türck, v. Graefe, Virchow, H. Müller, Traube, Förster, Wedl, Schweigger, Hutchinson, Leber u. A.

2) Virchow, Zur pathologischen Anatomie der Netzhaut und des Sehnerven. Dieses Archiv Bd. X. 1856.

s) Eìne ausführliche Abhandlung von mir über den normalen Bau der äusseren Körnerschicht, sowie über den Bau und Verlauf der Lymphräume in derselben wird năchstens unter dem Titel „Zur Lehre über den Bau der äusseren Körnerschicht der Netzbaut bel den verschiedenen Wirbelthieren“ in dem Arch. für mikroskop. Anat. Bd. 19 Heft 3 erscheinen. In 
ihr entfernt. Es entsteht $z$ wischen ihnen und der Memb. limit. in der ganzen Ausdehnung der Netzhaut ein freier, 0,015 bis 0,021 Linien weiter $\mathrm{Z}$ wischenraum. Eine genaue Untersuchung ergiebt übrigens, dass dieser Raum nicht ganz frei ist von Körnern, die Zahl derselben ist aber an dieser Stelle nur sebr gering und ihre Entfernung von einander noch dazu äuserst unregelmässig; wir konnten an manchen Stellen die Entfernung zweier benachbarter Körner von einander auf 0,012 bestimmen, während an anderen Stellen ihre gegenseitige Entfernung von einander kaum 0,006 betrug. Der Grund dieser Unregelmässigkeit liegt darin, dass die in dem freien Zwischenraum noch anzutreffenden Körner Zapfenkörner sind, welche sich an ibren Zapfen halten müssen; die Zapfen selbst aber sind an den verschiedenen Stellen der Netzhaut verschieden weit von einander entfernt, wodurch auch die unregelmässige Entfernung jhrer Körner von einander bedingt wird. Den freien Zwischenraum fanden wir in sämmtlichen Netzhäuten der an Morbus Brightii verstorbenen Personen, gleichviel, ob die Krankheit grössere oder geringere Veränderung hervorgerufen hatte. An einer und derselben Netzhaut war die Weite des Zwischenraumes ganz unabbängig von der Grösse der Anschwellung; wir sahen diesen Zwischenraum sogar an solchen Stellen noch sehr gut, wo wir an der Netzhaut gar keine Anscbwellung wahrnehmen konnten. Ebenso wenig wird die Weite des freien Zwischenraumes von der grösseren oder geringeren Zahl der Zapfen an dieser Stelle beding ${ }^{1}$ ). Von entscheidender Wichtigkeit für die Weite des Zwischenraumes ist dagegen die Stelle, die man zur Untersuchung wählt. Die Weite des Zwischenraumes ist in der Umgebung der Macula lutea am grössten, von da nimmt sie nach der Peripherie zu immer mehr ab und in der Gegend des Corpus ciliare ist der Zwischenraum kaum erkennbar.

Im normalen Zustande liegen die Körner sehr nahe neben einander, nur durch eine $Z$ wischensubstanz von minimaler Dicke von einander getrennt; die Lympbräume sind so schmal, dass man sie

demselben Archir wird dann eine zweite Abhandlnng Ueber den Bau und die Function des Kammes in der Netzhaut der Vögel folgen, worauf ich die geehrten Leser hier aufmerksam mache.

1) Die Fovea centralis muss von dieser Regel ausgenommen werden. Hier ist der Zwischenraum eng, da sämmtliche Körner hier Zapfenkörner sind und der Memb. limit. năher liegen, wie wir es weiter unten erörtern werden. 
nur bei starker Vergrösserung und an feinen Schnittpräparaten sehen kann. Im pathologischen Zustande sieht man schon an groben Präparaten die ganze äussere Körnerschicht durch schmale, von der Memb. limit. nach innen ziehende Furchen in Abtheilungen getheilt. Diese Furchen erweisen sich bei sorgfältigerer Untersuchung als die stark erweiterten Lymphräume dieser Schicht; ihr Durchmesser hat sich unter dem. Einfluss des paihologisches Prozesses von 0,006 Linien auf $0,018-0,021$ Linien vergrössert. Sämmtliche Lymphräume in dieser Schicht münden in den freien Raum, den wir eben als zwischen der Memb. limit. ext. und den Körnern verlaufend beschrieben haben. Die solchermaassen erweiterten Lymphräume sind in der ganzen Ausdehnung der Netzhaut fast bis zum Corpus ciliare anzutreffen, wie wir es an einem Meridianschnitt sehen konnten. Auch die Körner rücken in diesem Zustande der Netzhaut weiter auseinander. Sie erscheinen unter dem Mikroskop wie in Unordnung über das Gesichtsfeld hingestreut, wie man es auf der Abbildung, sehen kann (Fig. 2).

Die centralen Fortsätze der Körner in dieser Schicht besitzen im normalen Zustande an verschiedenen Stellen der Netzhaut eine ungleiche Länge. Sie bilden in der Netzhaut eine besondere Schicht (M. Schultze'sche Faserschicht), deren Dicke je nach der betreffenden Stelle verschieden sein kann. So besitzt sie in der Gegend des Corpus ciliare kaum eine Dicke von 0,006-0,009 Linien, ihre Dicke nimmt aber immer mehr zu, je näher dem Centrum der Netzhaut, und in der Nähe der Macula lutea hat sie allmählich, ohne dass man schroffe Uebergänge sieht, eine Dicke von 0,090 Linien erreicht. Die Dicke dieser Schicht hängt direct von der Länge und Richtung der einzelnen Fortsätze ab. An der Peripherie der Netzhaut sind die Fortsätze gewöhnlich kurz, sie werden immer länger, je näher am Centrum sie verlaufen; mit der Länge ändern sie auch ihre Richtung; während nehmlich die kurzen, in der Nähe des Corpus ciliare verlaufenden Fortsätze senkrecht zur Zwischenkörnerschicht stehen, nehmen die Fortsätze nach der Gegend der Macula hin, wenn sie eine gewisse Länge erreicht haben, eine etwas schiefe Richtung an. Endlich treffen wir auf Fortsätze, welche ganz horizontal, beinahe parallel mit der Memb. limit. verlaufen, das sind auch die allerlängsten. Uebrigens sind die Fortsälze auch bei verschiedenen Personen verschie- 
den; die Länge und Richtung kann bei verschiedenen Individuen variiren.

An den Stellen, wo die Fortsätze noch senkrecht zur Zwischenkörnerschicht verlaufen, kann man zwischen ihnen die Lymphräume gut erkennen; man sieht sie unmittelbar in die Lymphräume der äusseren Körnerschicht übergehen, sowie vermittelst schmaler Spalten mit den Räumen in der inneren Körnerschicht communiciren. Auch da, wo diese Fortsätze schon eine elwas schiefe Richtung zur Zwischenkörnerschicht haben, kann man noch die Lymphräume verfolgen, an den Stellen aber, wo die schiefe Richtung der Fortsätze eine gewisse Grenze überschritten hat, sind die Lymphräume nur mit grosser Mühe zu verfolgen; nur in dem, der Zwischenkörnersehicht anliegenden Theile kann man noch Räume von unregelmässiger Form erkennen, die sich zwischen den centralen Fortsätzen verlieren ${ }^{1}$ ).

Im pathologischen Zustande sind die Lymphräume zwischen den centralen Fortsätzen mehr entwickelt, ihre Verbindung mit denselben Räumen in der äusseren, sowie in der inneren Körnerschicht ist viel deutlicher ausgesprochen. Die Hauptveränderung sehen wir aber an den Stellen der Netzhaut, wo die centralen Fortsätze eine stark geneigte Richtung zur Zwischenkörnerschicht annehmen. Die Netzhaut ist hier stark verdickt. Die Verdickung geschieht auf Kosten der schiefen Fortsätze, indem ein Theil von ihnen sich aufrichtet und sich senkrecht zur Zwischenkörnerschicht stellt. Da aber nicht alle solche centralen Fortsätze sich senkrecht zur Zwischenkörnerschicht stellen, sondern ein Theil von ihnen die Richtung, welche sie im normalen Zustande haben, beibehalten, so stossen wir hier auf die extremsten Dickenunterschiede, wie man es in der Fig. 3 sehen kann. Wir sahen nicht selten auf einem kleinen Raum von kaum $0,009-0,015$ die Dickendurchmesser von $0,03-0,05$ Linien auf $0,15-0,25$ Linien steigen. Im normalen Zustande findet der Uebergang nur ganz allmählich statt; bei der Bright'schen Krankheit fehlt dagegen diese langsame $\mathrm{Zu}$ - und Abnahme der Dicke. Darin und in der ungewöhnlichen Zunahme der Dicke sehen wir

1) Merkel stellt in seinen Abbildungen diesen, eben beschriebenen Theil der Netzhaut als ein Netz von Filzfasern dar. Ein solches Bild erhält man aber unter dem Mikroskop nur dann, wenn man die centralen Fortsätze quer durchschneidet. 
die Abweichung vom normalen Zustande. Die hier vorkommenden Lymphräume nehmen gleichfalls einen graden Verlauf an und erscheinen infolge dessen als weite Gänge von $0,015-0,021$ Linien, welche unmittelbar in die Räume der äusseren Körnerschicht übergehen.

Die gleichen Veränderungen, wie bei dem Morbus Brightii, fanden wir auch in der äusseren Körnerschicht bei anderen Krankheiten vor. An der äasseren Körnerschicht eines jungen Mannes, der zwar keine Spur von der Bright'schen Nierenkrankheit zeigte, dafür aber sonst mehrere Blutextravasate in der Netzhaut hatte, saben wir alle Veränderungen wieder, die wir beim Morbus Brightii beschrieben haben, wenn auch die Veränderungen in diesem Falle nicht so stark ausgesprochen waren. In einem dritten Falle war der 0pticus durch einen Gehirntumor gedrückt, hier konnten wir nur die Erweiterung der Lymphräume und den freien Zwischenraum zwischen der Memb. limit. ext. und den Körnerlagen constatiren. Die Lagenveränderung der centralen Fortsätze und die dadurch entstandene Verdickung dieser Schicht konnten wir nicht finden.

Alle diese Erscheinungen lassen sich durch den erhöhten Blutdruck in den Gefässen und den vermehrten Austritt von Flüssigkeit in das sie umgebende Gewebe gut erklären. Die Erweiterung der Lymphräume und die Entfernung der Körner von einander erklären sich aus dem erhöhten Druck in diesen Räumen ohne Weiteres, schwieriger ist die Entstehung des freien Zwischenraums zwischen den Körnerlagen und der Memb. limit. zu verstehen, indessen lässt sich auch diese Erscheinung aus den obigen Gründen erklären, wenn man die Verbindungsweise der Körner mit ihren Stäbchen und Zapfen berücksichtigt. Die Stäbchenkörner verbinden sich durch ihre sehr feinen peripherischen Fortsätze mit den Stäbchen, während die Zapfenkörner mit den Zapfen vermittelst der dicken Innenglieder der letzteren in Verbindung stehen. Wird nun durch irgend eine Ursache der Druck in den Lymphräumen der äusseren Körnerschicht erböht, so müssen einerseits die nachgiebigen peripherischen Fortsätze der Stäbchenkörner sich dehnen oder zerreissen, während andererseits die Memb. limit. im Ganzen etwas nach aussen gedrängt wird; dadurch muss zwischen den Stäbchenkörnern und der Memb. limit. ein Raum entstehen. Die dicken Innenglieder der Zapfen geben dem Druck weniger nach und werden nur wenig aus- 
gedehnt. Sie stehen auch nicht sehr viel weiter von der Memb. limit. als im normalen Zustande ab und finden sich auch in dem entstandenen Zwischenraum vor. Der erhöhte Druck ruft auch die Veränderung der Richtung der centralen Fortsätze und die Verdickung der Netzhaut hervor. Dass die Anschwellung eine wellenförmige ist, hängt erstens von der An- oder Abwesenheit von Blutgefässen in der betreffenden Stelle, dann von den grösseren oder geringeren Schwierigkeiten, die dem Rückfluss der Ernährungsflüssigkeit zu den Venen entgegenstehen, ab. Dass die Blutgefässe bei der Verdickung der Netzhaut eine hervorragende Rolle spielen, geht daraus hervor, dass die Anschwellung immer nur an der Stelle, wo unter dem äusseren Plättchen der Zwischenkörnerschicht ein Blutgefäss verläuft, eine ansehnliche Höhe erreicht. Untersuchen wir an einem genau senkrecht zur Oberfläche der Netzhaut geführten Schnitt eine einzelne, kleine Anschwellung von abgerundeter Form, so sehen wir die Lymphräume, welche senkrecht zu der Richtung eines Gefässes verlaufen, bedeutend erweitert und in grader Richtung verlängert, während seine Nachbarräume viel weniger vergrössert sind. Die stark erweiterten und verlängerten Lymphräume entsprechen der Spitze oder dem Höhepunkt der Anschwellung, die weniger erweiterten Räume entsprechen der Basis derselben. Die grössere oder geringere Anschwellung hängt also von der grösseren oder geringeren Ausdehnung der Lymphräume, diese aber ihrerseits von der Näbe der Blutgefässe ab. Der letztere Umstand ist für die Füllung der Lymphräume sehr wichtig, weil mehrere Räume ihre Lymphe von einem einzigen Blutgefäss beziehen; ein in der Nähe dieses Gefässes verlaufender Lymphraum muss seine Lymphe auf kürzerem Wege und schneller beziehen, als ein etwas entfernterer. Der erstere Raum wird demzufolge sich auch rascher und stärker ausdehnen, als die anderen Räume. Hatte die geschwollene Stelle der Netzhaut nicht die beschriebene runde Form und besass sie eine grössere Ausdehnung, so waren an dieser Stelle entweder mebrere Gefässe, oder ein Gefäss begleitete die Anschwellung in ihrer ganzen Länge. Das Verhalten der Lymphräume war dabei genau so, wie in dem ersteren Falle. Die Einwirkung der verhinderten Rückströmung der Ernährungssäfte wollen wir am Schluss dieser Abhandlung erörtern.

Wir wollen jetzt noch die Veränderungen besprechen, die 
sich an der äusseren Körnerschicht der Netzhaut eines Erhängten fanden. Dieses Auge hatte ich als . schon zum Untersuchen fertiges Präparat von Herrn Professor Waldeyer erhalten. Dasselbe war in ganz frischem Zustande durch einen grossen Schnitt durch die Cornea eröffnet und erst mit einer 1procentigen Ueberosmiumsäurelösung, dann mit Spiritus behandelt. An einem feinen Meridianschnitt findet man die Netzhaut in der Peripherie nur wenig verändert; je näher aber dem Centrum, desto schärfer treten uns die Veränderungen entgegen. Die Memb. limit. ist in der Gegend der Ora serrata wenig verdickt, man bemerkt aber bei starker Vergrösserung schon hier ganz feine Räume in ihrer Substanz, die immer grösser erscheinen, je näher zum Centrum des Auges wir untersuchen. Gleichzeitig nehmen sie eine abgerundete Form an. Bis zu dieser Stelle sind die Räume von allen Seiten von der Substanz der Membran eingeschlossen, weiter nach dem Centrum zu verwandeln sich die schmalen Spalten in runde oder ovale Gänge, die über die Memb. limit. hinaus nach aussen ragen; nach innen treten diese Räume mit den Lymphräumen in der äusseren Körnerschicht in Verbindung. In der Nähe der Macula lutea erweitern sie sich bis auf 0,018 Linien bei einer Länge von 0,015 Linien, manchmal vereinigen sich zwei solche Räume zu einem einzigen, 0,024 Linien weiten Raum. Die Entstehung und die Erweiterung dieser Räume geschieht auf Kosten der Umhüllungshäute der Zapfen, die in dièsem Falle so erweitert werden, dass sie ihren Zapfen wie eine Nachtmütze aufsitzen; manchmal fehlt einem Zapfen das ihm gehörige Korn, wobei die Umbüllungshaut die Form einer Flasche hat (Fig. 2) ${ }^{1}$ ). In der äusseren Körnerschicht selbst treten Veränderungen ein, die im Allgemeinen den oben beschriebenen sehr ähnlich sind. Die Lymphräume erweitern sich bis auf 0,021. Durch die Erweiterung der Lymphräume rückt ihre Wandung auseinander,

1) M. Schulze und Schwalbe geben an, dass der innere Theil der Zapfen in einem, aus Bindegewebsfasern bestehenden Körbchen liegt, während der äussere Theil keine Hülle habe. Wäre diese Angabe richtig, so müsste doch bet den beschriebenen Veränderungen, die auf einen, in der äusseren Körnerschicht stattgefundenen, starken Druck von Flüssigkeit deuten, diese Körbchen dem Drucke nachgeben, stellenweise zerreissen und die Flüssigkeit jenseits der Memb. limit. gleiten lassen. Da dieses jedoch keineswegs geschieht, so muss ich die Wahrheit dieser Angabe bezweifeln. 
wodurch auch die Körner, die eben diese Wandung bilden, mehr von einander entfernt erscheinen, als im normalen Zustande. Auch den Zwischenraum, den wir schon beim Morbus Brightii zwischen den Körnerlagen und der Memb. limit. ext. kennen gelernt haben, fanden wir hier wieder. Derselbe batte eine Weite von 0,006 bis 0,009 Linien und war fast ganz frei, nur hie und da sahen wir die peripherischen Fortsătze der Stäbchenkörner, die zu den Stäbchen durch diesen Raum ziehen, oder einige seltene Zapfenkörner.

Aus den Experimenten von Ranvier, die auch von Rott wiederholt wurden, wissen wir, dass nach Unterbindung von Venenstämmen, gleichviel, ob die Nerven erst durchschnitten wurden oder nicht, das Gewebe ödematös wird, d. h. wenn inzwischen kein collateraler Kreislauf entsteht, schwellen die Lymphgefässe und Lymphräume, welche bisher wegen ihrer Kleinbeit unsichtbar waren, so an, dass man sie gut sehen kann. Der Grund dër Erscheinung beruht darauf, dass die Nährstoffe aus den Geweben in die Lymphräume aufgenommen werden und nicht mehr aus diesen nach den Venen strömen können, wodurch die Lymphräume überfüllt und stark erweitert werden. Dieselbe Erscheinung sahen wir auch in der Netzhaut.

Ueberblicken wir die pathologischen Veränderungen in sämmtlichen, von uns beschriebenen Fällen, so seben wir, dass alle diese Erscheinungen in der letzten Instanz durch eine und dieselbe Ursache, durch den behinderten Abfluss des Blutes aus der Netzhaut, hervorgerufen werden. Auf welche Ursachen diese Stockung des Abflusses auch zurückzuführen ist, immer hat sie dieselben. Veränderungen in der äusseren Körnerschicht zur Folge. Dass allen diesen Fällen wirklich die Stockung des Abflusses gemeinschaftlich ist, lässt sich unschwer beweisen. Beim Morbus Brightii sind die Gefässwände verdickt, das Gefässlumen verengt, der 0pticus ist mit Flüssigkeit getränkt, sein Gewebe aufgequollen. Dieselbe Veränderung ist auch mit der Papilla $n$. optici vorgegangen [Virchow ${ }^{1}$ )]. Dadurch wird aber ein Druck auf die Vena centralis retinae ausgeübt und zu den sonstigen Kreislaufshindernissen kommt noch der mechanische Druck hinzu. In dem Fall eines Tumor am Grosshirn

1) Virchow, Zur pathologischen Anatomie der Netzhant und des Sehnerven. Dieses Archiv Bd. X. 1856 . 
wurde ein starker Druck auf den im Schädel verlaufenden Theil des Opticus ausgeübt, der sich auf die Vena ophtbalmica fortpflanzte. Bei einem Erhängten traten die Erscheinungen der Stockung des Abflusses im ganzen Kopftheil auf. Diese Erscheinungen treten sehr schnell ein, woraus sich die grossen Veränderungen in der Membrana limitans externa erklären lassen.

\section{Erklärung der Abbildungen.}

Taf. lX. Fig. $1-3$.

Fig. 1. Veränderungen in der äusseren Körnerschicht und der äusseren Faserschicht beim Morbus Brighti in der Gegend der Macula lutea bei starker Vergrösserung.

Fig. 2. Verănderung der Membrana limitans externa und der äusseren Körnerschicht des Erhängten. s z Zapfen und Stäbchenschicht. le Membrana limitans externa. a k Aeussere Körnerschicht. c f Aeussere Faserschicht (Schicht der centralen Fortsätze der Körner) der äusseren Körnerschicht. i $k$ Innere Körnerschicht. m Molecularschicht. g Ganglienzellenschirht. p Freier Zwischenraum zwischen der Membrana limitans externa und dem Körnerlager. q Peripherischer Fortsatz der Körner der äusseren Körnerșchicht. o Ein erweiterter Lymphraum in der äusseren Körnerschicht. $k$ Erweiterung der Umhüllungsmembran der Zapfen. $r$ Gefässe.

Fig. 3. Dasselbe, wie in Fig. 1, bei geringerer Vergrösserung. 


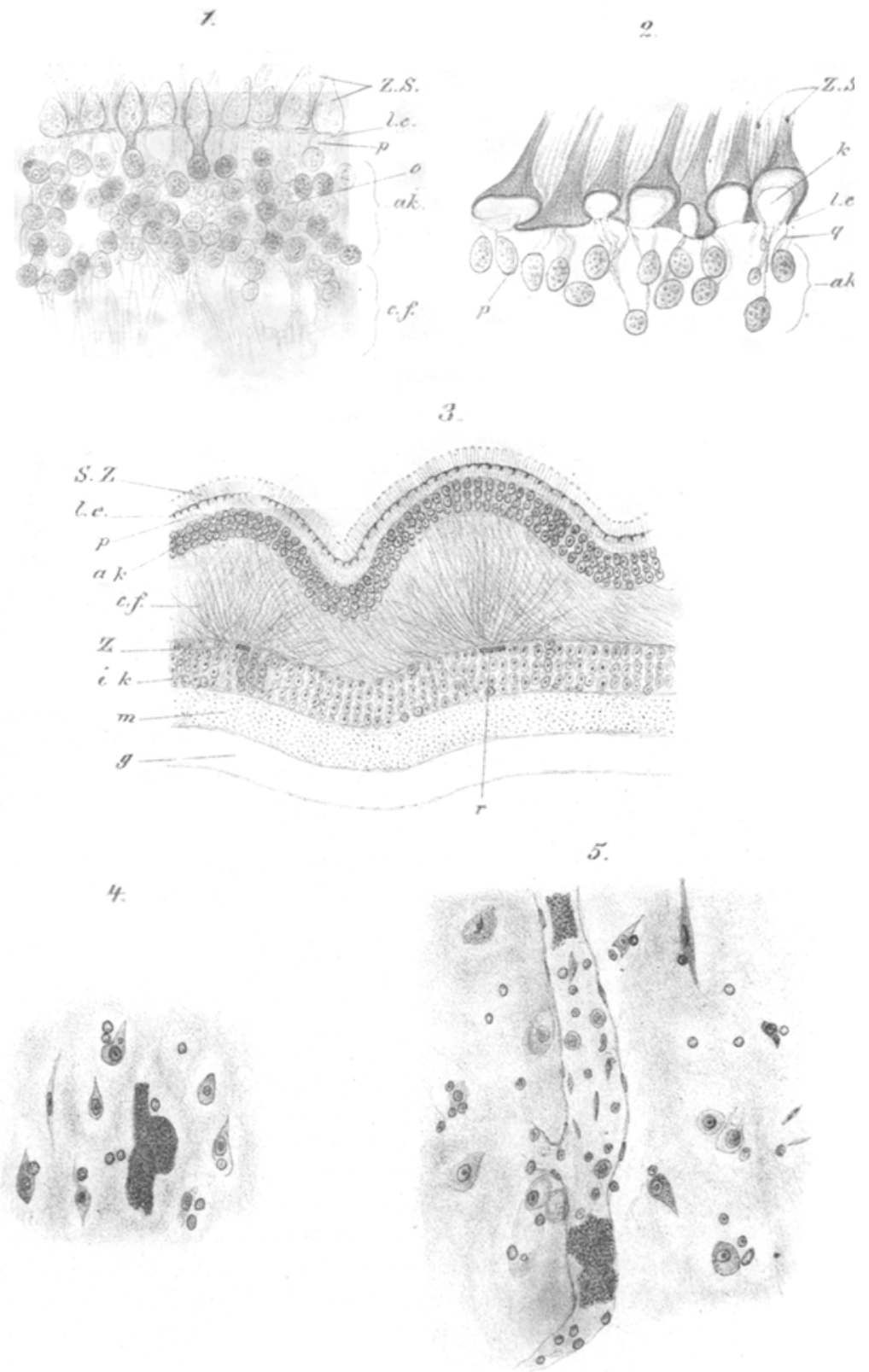

elle. Schinze Sich. Onst. Borlin. 